\title{
MANIFOLDS IN WHICH THE POINCARÉ CONJECTURE IS TRUE
}

\author{
BY \\ JONATHAN L. GROSS $\left({ }^{1}\right)$
}

1. Introduction. A homotopy 3-cell is a compact, connected triangulated 3manifold whose boundary is a 2-sphere and whose fundamental group is trivial. The Poincaré conjecture is true in dimension three if and only if every homotopy 3-cell is a 3-cell. If $M$ is a 3-manifold, one says that the Poincaré conjecture is true in $M$ provided that every imbedded homotopy 3-cell in $M$ is a 3-cell. Equivalently, one says that $M$ is in PC (the "Poincaré Category").

It will be shown in this paper that whenever any 3-manifold in PC is pasted to itself or to any other 3-manifold in PC across a disk, a sphere, or a projective plane, the resulting 3-manifold is in PC. Moreover, it will be shown that if pasting across any compact 2-manifold other than a disk, a sphere, or a projective plane always preserves membership in PC, then the Poincaré conjecture is true.

It follows from Theorem 1 of Moise [6] that if the underlying space of a homotopy 3-cell can be topologically imbedded in a 3-manifold $M$, then there is a piecewise linear imbedding of that homotopy 3-cell into any triangulation of $M$ (and, of course, $M$ can be triangulated). Therefore, if every piecewise linearly imbedded homotopy 3-cell in any particular triangulation of $M$ is a 3-cell, then $M$ is in PC.

From now on, any manifold considered will be triangulated, and any map considered will be piecewise linear.

2. Examples of 3-manifolds in PC. Explicit consideration of PC probably begins with Brown [1], in which these four examples are given.

(2.1) Euclidean 3-space $R^{3}$ is in PC.

(2.2) If $B$ is a 3-manifold in PC, then every 3-dimensional submanifold of $B$ is in $\mathrm{PC}$.

(2.3) If a 3-manifold $B$ has a covering space which is in PC, then $B$ is in PC.

(2.4) If $M$ is a 2 -manifold, then the 3 -manifold $M \times[0,1]$ is in PC.

The following examples further indicate the extent of PC.

(2.5) The 3-sphere $S^{3}$ is in PC.

Proof. This follows from Alexander's Theorem.

(2.6) The lens spaces are in PC.

Presented to the Society, January 24, 1969; received by the editors March 29, 1968.

(1) The author wishes to thank Professor Edward M. Brown for his help and encouragement. This work was partially supported by National Science Foundation Grant GP6526. 
Proof. The universal covering space of a lens space is a 3-sphere (see, for example, 6.7.5 of Hilton and Wylie [4]). By (2.5) and (2.3), therefore, the lens spaces are in PC.

(2.7) Let $M$ be a 2-manifold. Then the total space of any fibre bundle whose base space is the 1-sphere $S^{1}$ and whose fibre is $M$ is in PC.

Proof. Such a total space is covered by $M \times R^{1}$, which is imbeddable in $M \times I$. By (2.4) and (2.2), $M \times R^{1}$ is in PC. By (2.3), therefore, the total space is in PC.

(2.8) The connected sum of two compact, orientable, connected 3-manifolds in $\mathrm{PC}$ is also in PC.

Proof. This follows from Theorem 1 of Milnor [5].

(2.9) If each of two compact, orientable connected 3-manifolds with connected nonvacuous boundary is in PC, then the 3-manifold obtained by pasting them together across a disk is also in PC.

Proof. This follows from the Decomposition Theorem of the author [2].

Example (2.9) is a special case of the present Theorem 1 of $\S 4$.

3. $P$-admissibility. The 3-manifolds considered in this paper are, in general, not connected. Thus, any result concerning the operation of pasting a 3-manifold to itself will also apply to the operation of pasting two disjoint 3-manifolds together.

Convention. Let $B$ be a 3-manifold which is obtained by pasting a 3-manifold $M$ (connected or not) to itself across two disjoint homeomorphic 2-dimensional submanifolds of its boundary. It will always be assumed that the triangulation of $B$ is sufficiently fine that the identification map $M \rightarrow B$ (which is induced by the pasting operation) is simplicial for some subdivision of the triangulation of $M$. In particular, the image in $B$ of the pasting surfaces is assumed to be simplicially imbedded in $B$.

Definition. Let $R$ be a compact connected 2-manifold. One says that $R$ is $P$-admissible if the following holds: For any 3-manifold $B$ (connected or not) in PC and for any pair of imbeddings $f: R \rightarrow$ bd $(B)$ and $g: R \rightarrow$ bd $(B)$ whose images are disjoint, the 3-manifold obtained from $B$ by identifying, for each point $x$ in $R$, the points $f(x)$ and $g(x)$ of bd $(B)$ is in PC.

The main result of this paper may now be formulated as follows.

PASTING THEOREM. The disk, the sphere, and the projective plane are P-admissible. Furthermore, if any other compact connected 2-manifold is P-admissible, then the Poincaré conjecture is true.

4. Disks are $P$-admissible. The sole purpose of this section is the proof of Theorem 1. The numbered statements $(4.1), \ldots,(4.6)$ are intermediate results in that proof.

THEOREM 1. Let $M$ be a 3-manifold (connected or not) in PC. Let $D^{\prime}$ and $D^{\prime \prime}$ be disjoint disks on bd $(M)$. And let $B$ be a 3-manifold obtained from $M$ by identifying $D^{\prime}$ and $D^{\prime \prime}$ under a homeomorphism. Then $B$ is in $P C$. 
Proof. Let the disk $D$ be the image in $B$ of the disks $D^{\prime}$ and $D^{\prime \prime}$. If a given homotopy 3-cell can be imbedded in $B$, then there is an imbedding of that homotopy 3-cell such that its image $C$ lies in the interior of $B$ and such that the components of bd $(C) \cap D$ are simple loops, each a crossing of surfaces. The theorem will be proved by an induction on the number of these loops.

Basis step. If bd $(C) \cap D$ is void, then $C \subset B-D=M-\left(D^{\prime} \cup D^{\prime \prime}\right) \subset M$. Since $M$ is in PC, the homotopy 3-cell $C$ is a 3-cell.

Induction step. It will be shown that if bd $(C) \cap D$ has exactly $n$ components, then $C$ is a 3 -cell.

The regular neighborhood theory of Whitehead [8] implies the existence of a regular neighborhood of bd $(C)$ and a regular neighborhood of $D$ whose intersection is a regular neighborhood of bd $(C) \cap D$. The regular neighborhoods of bd $(C)$ and $D$ will be realized here as the images of a pair of homeomorphisms $F:$ bd $(C) \times[-1,1] \rightarrow B$ and $H: D \times[-1,1] \rightarrow B$ such that the following conditions hold (see Figure 1).

(i) For all points $x$ of bd $(C), F(x, 0)=x$.

(ii) $F$ (bd $(C) \times\{-1\}) \subset C$.

(iii) For all points $y$ of $D, H(y, 0)=y$.

(iv) $H($ bd $(D) \times[-1,1])=H(D \times[-1,1]) \cap$ bd $(B)$.

(v) $F($ bd $(C) \times[-1,1]) \cap H(D \times[-1,1])=$

$$
H(F((\text { bd }(C) \cap D) \times[-1,1]) \times[-1,1]) .
$$

In order to facilitate cutting and pasting the following additional condition is imposed.

(vi) For all points $x$ of bd $(C) \cap D$ and all points $u, v$ of $[-1,1], F(H(x, u), v)$ $=H(F(x, v), u)$.

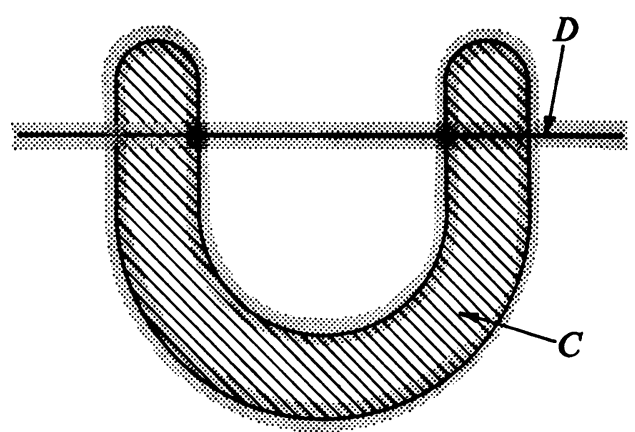

FIGURE 1

(4.1) If any component of $C \cap D$ is a disk, then $C$ is a 3-cell.

Proof of (4.1). Let $E$ be a component of $C \cap D$ which is a disk. The components of $C-H(E \times(-1,1))$ are homotopy 3-cells, by the van Kampen theorem. There are fewer than $n$ component loops in the intersection of the boundary of either component of $C-H(E \times(-1,1))$ with the disk $D$. Therefore, by the induction 
hypothesis, both components are 3-cells. Hence, $C$ is a 3-cell (by Theorem 6 on p. III-19 of Zeeman [9], for example).

Definition. Let $M$ and $N$ be manifolds, and let $f: M \rightarrow N$ be an imbedding. One says that $f$ is a proper imbedding if $f(\mathrm{bd}(M)) \subset$ bd $(N)$ and $f(\operatorname{int}(M)) \subset \operatorname{int}(N)$.

The purpose of making the definitions of the next few paragraphs is to split the homotopy 3-cell $C$ into two pieces, which will be called $K_{0}$ and $K_{0}^{\prime}$. The rest of the proof of the theorem consists of first showing that $K_{0} \cap K_{0}^{\prime}$ is a disk, properly imbedded in $C$ (which implies, by the van Kampen theorem, that $K_{0}$ and $K_{0}^{\prime}$ are homotopy 3-cells), and then showing that $K_{0}$ and $K_{0}^{\prime}$ are 3-cells.

Because of statement (4.1), it will henceforth be assumed that no component of $C \cap D$ is a disk. Thus the components of $C \cap D$ are all disks-with-holes. Each such disk-with-holes is contained in a minimal subdisk of $D$. An innermost component of $C \cap D$ on the disk $D$ is a component such that the minimal subdisk which contains it contains no other component of $C \cap D$.

Let $D_{0}$ be an innermost component of $C \cap D$ on the disk $D$. Let $l_{0}, \ldots, l_{m}$ be the components of bd $\left(D_{0}\right)$, with the loop $l_{0}$ outside of the loops $l_{1}, \ldots, l_{m}$ on $D$.

For $j=1, \ldots, m$, let $E_{j}$ be the disk on bd $(C)$ such that bd $\left(E_{j}\right)=l_{j}$ and $l_{0} \notin E_{j}$. One assumes that the loops $l_{1}, \ldots, l_{m}$ are indexed so that if $j<i$, then the number of disks among $E_{1}, \ldots, E_{m}$ which contain $E_{j}$ is not greater than the number of disks among $E_{1}, \ldots, E_{m}$ which contain $E_{i}$. Thus, the first few disks (i.e. those with lowest indices) are maximal as sets among $E_{1}, \ldots, E_{m}$. Each of the next few disks is contained by exactly one member of the previous batch, and so on.

Since $D_{0}$ is a cycle in $C_{2}\left(C\right.$, bd $\left.(C) ; Z_{2}\right)$, it must also be a boundary, because the homology of $C$ is trivial. Therefore, $C-D_{0}$ has two components, whose closures will be denoted by $K_{m}$ and $K_{m}^{\prime}$, with $K_{m}$ chosen so that it contains $H\left(D_{0} \times[0,1]\right)$. For $j>0$, given $K_{j}$, one defines $K_{j-1}=K_{j} \cup F\left(E_{j} \times[-j / n, 0]\right)$ if $H\left(l_{j} \times[-1,0]\right) \subset E_{j}$, or $K_{j-1}=K_{j}-F\left(E_{j} \times(-j / n, 0]\right)$ if $H\left(l_{j} \times[0,1]\right) \subset E_{j}$. Also, for $j>0$, given $K_{j}^{\prime}$, one defines $K_{j-1}^{\prime}=K_{j}^{\prime}-F\left(E_{j} \times(-j / n, 0]\right)$ if $H\left(l_{j} \times[-1,0]\right) \subset E_{j}$, or $K_{j-1}=K_{j}^{\prime} \cup F\left(E_{j} \times[-j / n, 0]\right)$ if $H\left(l_{j} \times[0,1]\right) \subset E_{j}$ (see Figure 2).

For $j=0, \ldots, m-1$, define $R_{j}$ to be the union of the disk-with-holes

$$
\left.D_{0}-\left(F\left(l_{j+1} \times(-(j+1) / n, 0]\right)\right) \cup \cdots \cup F\left(l_{m} \times(-m / n, 0]\right)\right)
$$

with the disks $F\left(E_{j+1} \times\{-(j+1) / n\}\right), \ldots, F\left(E_{m} \times\{-m / n\}\right)$. Also, define $R_{m}=D_{0}$.

(4.2) For $j=0, \ldots, m, R_{j}$ is a disk-with-j-holes.

Proof of (4.2). First of all, $R_{m}$ is a disk-with- $m$-holes. because $R_{m}=D_{0}$. Now for a given positive index $j \leqq m$, suppose that $R_{j}$ is a disk-with-j-holes. Since bd $(C) \cap D_{0}$ $=l_{0} \cup \cdots \cup l_{m}$ and $E_{j} \subset$ bd $(C)-l_{0}$, it follows that $E_{j} \cap D_{0} \subset l_{1} \cup \cdots \cup l_{m}$. If $l_{i} \subset E_{j}$, then $E_{i} \subset E_{j}$, by definition of the disks $E_{i}$. Thus by the indexing criterion for the disks $E_{i}, E_{j} \cap D_{0} \subset l_{j} \cup \cdots \cup l_{m}$. It follows that the intersection of the disk $F\left(E_{j} \times\{-j / n\}\right)$ with $D_{0}-\left(F\left(l_{j} \times(-j / n, 0]\right) \cup \cdots \cup F\left(l_{m} \times(-m / n, 0]\right)\right)$ is the loop $F\left(l_{j} \times\{-j \mid n\}\right)$. Evidently, the intersection of $F\left(E_{j} \times\{-j / n\}\right)$ with each of the disks $F\left(E_{j+1} \times\{-(j+1) / n\}\right), \ldots, F\left(E_{m} \times\{-m / n\}\right)$ is void. Therefore, the intersection of 

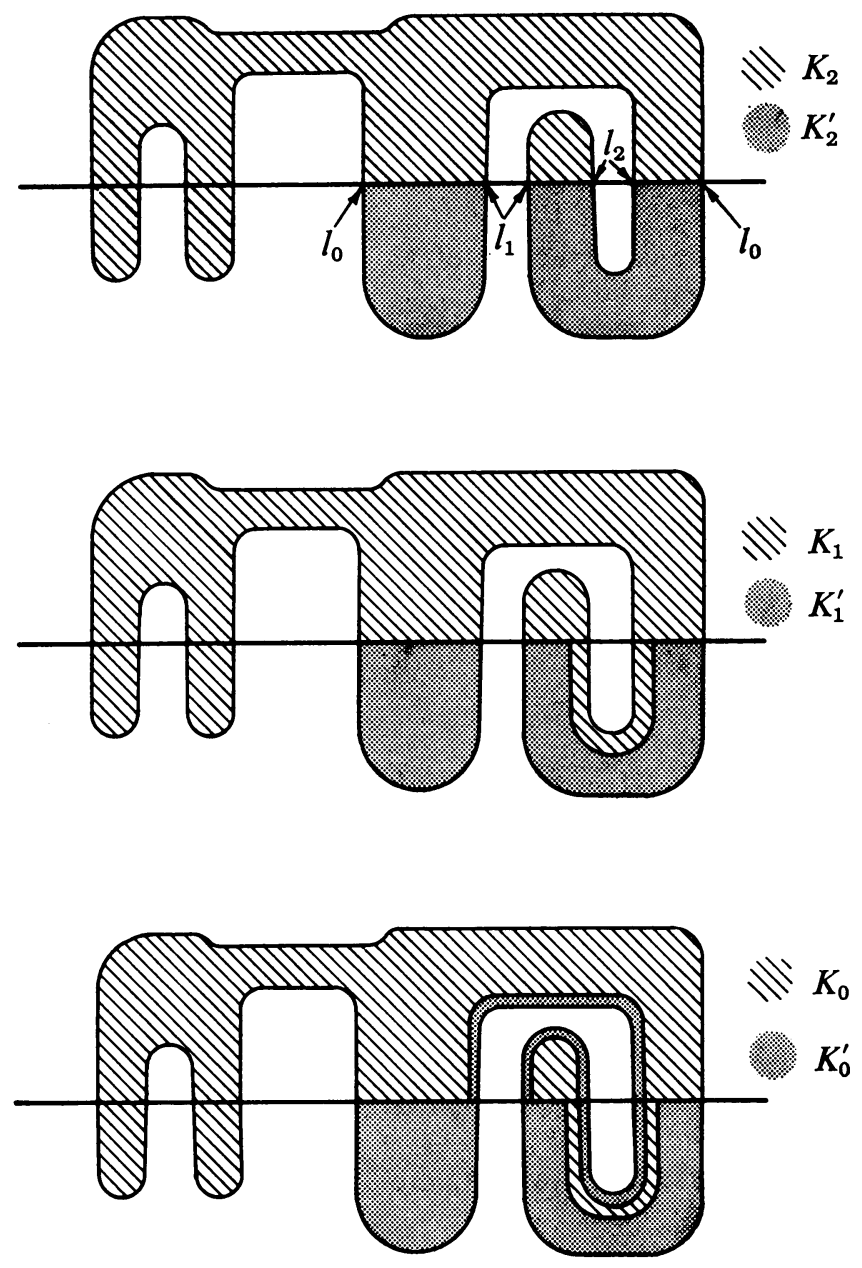

Figure 2

the disk $F\left(E_{j} \times\{-j / n\}\right)$ with $R_{j}-F\left(l_{j} \times(-j / n, 0]\right)$ is the loop $F\left(l_{j} \times\{-j / n\}\right)$. Since $R_{j}$ is a disk-with-j-holes, so is $R_{j}-F\left(l_{j} \times(-j / n, 0]\right)$. Since $R_{j-1}$ is the union of $F\left(E_{j} \times\{-j / n\}\right)$ and $R_{j}-F\left(l_{j} \times(-j / n, 0]\right)$, it must be a disk-with- $(j-1)$-holes.

(4.3) For $j=0, \ldots, m, K_{j} \cap K_{j}^{\prime}=R_{j}$.

Proof of (4.3). Since $K_{m}$ and $K_{m}^{\prime}$ are the closures of the two components of $C-R_{m}$, it follows that $K_{m} \cap K_{m}^{\prime}=R_{m}$. Now for a given positive index $j \leqq m$, suppose that $K_{j} \cap K_{j}^{\prime}=R_{j}$. There are two cases, depending upon whether $H\left(l_{j} \times[-1,0]\right)$ $\subset E_{j}$ or $H\left(l_{j} \times[0,1]\right) \subset E_{j}$. The symmetry of the definitions for $K_{j}$ and $K_{j}^{\prime}$ assures one that it is sufficient to consider only one of these cases. So suppose that $H\left(l_{j} \times[-1,0]\right) \subset E_{j}$. From the indexing condition on the disks $E_{1}, \ldots, E_{m}$ it follows that some collection $E_{j+1}, \ldots, E_{r}$ contains all the disks among $E_{j+1}, \ldots, E_{m}$ which are maximal as sets among $E_{j+1}, \ldots, E_{m}$. One permutes the indices $j+1, \ldots, r$ so that for $i=j+1, \ldots, r, E_{i} \subset E_{j}$ if and only if $i \leqq s$. 
One observes that cl $\left(E_{j}-\left(E_{j+1} \cup \cdots \cup E_{s}\right)\right)$ is a disk-with-holes whose boundary components are the loops $l_{j}, \ldots, l_{s}$ and whose intersection with $R_{j}\left(=K_{j} \cap K_{j}^{\prime}\right)$ is the union of the loops $l_{j}, \ldots, l_{s}$. Since $H\left(l_{j} \times[-1,0]\right) \subset E_{j}$, it follows from the construction of $K_{j}^{\prime}$ that $\mathrm{cl}\left(E_{j}-\left(E_{j+1} \cup \cdots \cup E_{s}\right)\right)$ lies in $K_{j}^{\prime}$ and that $H\left(l_{j} \times[0,1]\right)$ $\subset K_{j}$. It will now be shown that $H\left(l_{j+1} \times[0,1]\right) \subset E_{j+1}$. Otherwise, $F\left(E_{j+1} \times\right.$ $[-j / n, 0]) \subset K_{j}$, from the construction of $K_{j}$. Let $b$ be a path in the interior of $E_{j}$ from a point in $E_{j}-\left(E_{j+1} \cup \cdots \cup E_{s}\right)$ to a point in the interior of $E_{j+1}$. By the construction of $R_{j}$, the path $b$ never crosses $R_{j}$. But it goes from a point in $K_{j}^{\prime}$ to a point in $K_{j}$. This is a contradiction. Similarly, for $i=j+2, \ldots, s, H\left(l_{i} \times[0,1]\right) \subset E_{i}$.

It now follows that for $i=j+1, \ldots, s$, the 3-cell $F\left(E_{i} \times[-i / n, 0]\right)$ lies in $K_{j}^{\prime}$. Therefore, $F\left(E_{j} \times\left[-\left(j+\frac{1}{2}\right) / n, 0\right]\right) \subset K_{j}^{\prime}$, which implies that $F\left(E_{j} \times[-j / n, 0]\right) \cap K_{j}$ $=F\left(l_{j} \times[-j / n, 0]\right)$. Hence

$$
\begin{aligned}
K_{j-1} \cap K_{j-1}^{\prime}= & \left(K_{j} \cup F\left(E_{j} \times[-j / n, 0]\right)\right) \cap\left(K_{j}^{\prime}-F\left(E_{j} \times(-j / n, 0]\right)\right) \\
= & \left(K_{j} \cap\left(K_{j}^{\prime}-F\left(E_{j} \times(-j / n, 0]\right)\right)\right) \cup\left(F\left(E_{j} \times[-j / n, 0]\right)\right. \\
& \left.\cap\left(K_{j}^{\prime}-F\left(E_{j} \times(-j / n, 0]\right)\right)\right) \\
= & \left(R_{j}-F\left(l_{j} \times(-j / n, 0]\right)\right) \cup F\left(E_{j} \times\{-j / n\}\right)=R_{j-1} .
\end{aligned}
$$

Thus, statement (4.3) is proved.

(4.4) $K_{0}$ and $K_{0}^{\prime}$ are homotopy 3-cells whose union is the homotopy 3-cell $C$ and whose intersection is the disk $R_{0}$, which is properly imbedded in $C$.

Proof of (4.4). By their constructions, the union of $K_{0}$ and $K_{0}^{\prime}$ is $C$. By statement (4.2), $R_{0}$ is a disk. By its construction, $R_{0}$ is properly imbedded in $C$. By statement (4.3), $K_{0} \cap K_{0}^{\prime}=R_{0}$. By the van Kampen theorem, $K_{0}$ and $K_{0}^{\prime}$ must be homotopy 3-cells.

It will soon be proved that $K_{0}$ and $K_{0}^{\prime}$ are 3-cells. For this, it will be convenient to define certain new objects.

For $j=1, \ldots, m$, let $D_{j}$ be the subdisk of $D$ such that bd $\left(D_{j}\right)=l_{j}$.

Let $L_{m}=K_{m}$ and let $L_{m}^{\prime}=K_{m}^{\prime}$. For $j>0$, given $L_{j}$, one defines $L_{j-1}=L_{j}$ $\cup H\left(D_{j} \times\left[0, \frac{1}{2}\right]\right)$ if $H\left(l_{j} \times[-1,0]\right) \subset E_{j}$ and $L_{j-1}=L_{j}$ if $H\left(l_{j} \times[0,1]\right) \subset E_{j}$. Also, for $j>0$, given $L_{j}^{\prime}$, one defines $L_{j-1}^{\prime}=L_{j}^{\prime}$ if $H\left(l_{j} \times[-1,0]\right) \subset E_{j}$ and $L_{j-1}=L_{j}^{\prime} \cup$ $H\left(D_{j} \times\left[-\frac{1}{2}, 0\right]\right)$ if $H\left(l_{j} \times[0,1]\right) \subset E_{j}$ (see Figure 3).

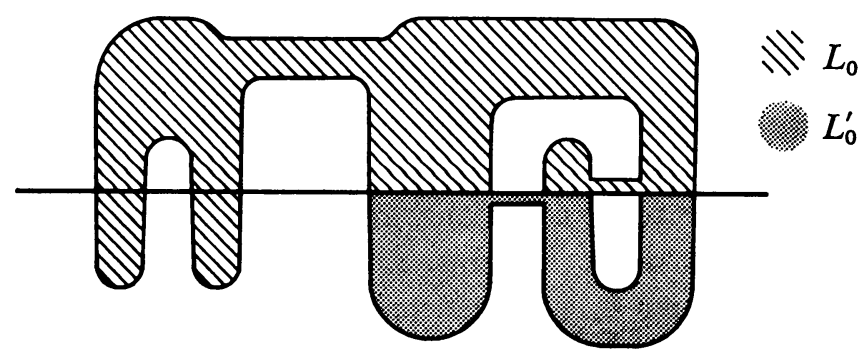

Figure 3 
(4.5) For $j=0, \ldots, m$, there is a homeomorphism $f_{j}: K_{j} \rightarrow L_{j}$. In particular, $K_{0}$ and $L_{0}$ are homeomorphic homotopy 3-cells.

Proof of (4.5). This proof will be accomplished by a lot of cutting and pasting operations, and the amount of detail is regrettably large. An examination of $K_{0}$, $K_{1}$, and $K_{2}$ in Figure 2 reveals that they are homeomorphic to $L_{0}, L_{1}$, and $L_{2}$, respectively, in Figure 3. In what follows the correspondence visible in the figures will be described rigorously.

For $1 \leqq i \leqq m$, for $y \in l_{i}$, for $-1 \leqq u \leqq 0$, and for $0 \leqq v \leqq 1$, let $h_{i}(F(H(y, v), u))$ $=(y, v, u)$ define a homeomorphism from $F\left(H\left(l_{i} \times[0,1]\right) \times[-1,0]\right)$ onto $l_{i} \times[0,1]$ $\times[-1,0]$, and let $g_{i}$ be the imbedding of $\left(l_{i} \times[0,1] \times\{-1\}\right) \cup\left(l_{i} \times\{0,1\} \times[-1,0]\right)$ into bd $\left(F\left(H\left(l_{i} \times[0,1]\right) \times[-1,0]\right)\right)$ which is given as follows: let $g_{i}(y, v,-1)$ $=F(H(y, v),-1)$, let $g_{i}(u, 1, u)=F(H(y, 1), u)$, if $-1 \leqq u \leqq-i / n$ then let $g_{i}(y, 0, u)$ $=F(H(y, 0),(n u+i) /(n-i))$, and if $-i / n \leqq u \leqq 0$ then let $g_{i}(y, 0, u)=F(H(y$, $(i+n u) / 2 i, 0)$. By the Product Theorem of Brown [1], each map $g_{i}$ extends to a homeomorphism of $l_{i} \times[0,1] \times[-1,0]$ onto $F\left(H\left(l_{i} \times[0,1]\right) \times[-1,0]\right)$, which will be denoted by $g_{i}^{\prime}$. It is clear that the autohomeomorphism $g_{i}^{\prime} h_{i}$ on $F\left(H\left(l_{i} \times[0,1]\right)\right.$ $\times[-1,0])$ takes $F\left(l_{i} \times[-i / n, 0]\right)$ onto $H\left(l_{i} \times\left[0, \frac{1}{2}\right]\right)$ and leaves $F\left(H\left(l_{i} \times[0,1]\right)\right.$ $\times[-1]) \cup F\left(H\left(l_{i} \times\{1\}\right) \times[-1,0]\right)$ fixed.

Define $f_{m}: K_{m} \rightarrow L_{m}$ to be the homeomorphism which is the identity on the complement in $K_{m}$ of the union of $F\left(H\left(l_{1} \times[0,1]\right) \times[-1,0]\right), \ldots, F\left(H\left(l_{m} \times[0,1]\right)\right.$ $\times[-1,0])$ and which is the map $g_{i}^{\prime} h_{i}$ on each of the solid tori $F\left(H\left(l_{i} \times[0,1]\right)\right.$ $\times[-0,1])$. For a positive index $j \leqq m$ suppose that a homeomorphism $f_{j}: K_{j} \rightarrow L_{j}$ has been defined so that if $1 \leqq i \leqq j$ then $f_{j}\left(F\left(l_{j} \times[-i / n, 0]\right)\right)=H\left(l_{j} \times\left[0, \frac{1}{2}\right]\right)$.

Suppose that $H\left(l_{j} \times[-1,0]\right) \subset E_{j}$. Then $K_{j-1}=K_{j} \cup F\left(E_{j} \times[-j / n, 0]\right)$ and $L_{j-1}=L_{j} \cup\left(D_{j} \times\left[0, \frac{1}{2}\right]\right)$. It was shown in the proof of statement (4.3) that $K_{j}$ $\cap F\left(E_{j} \times[-j / n, 0]\right)=F\left(l_{j} \times[-j / n, 0]\right)$. It is evident that $L_{j} \cap H\left(D_{j} \times\left[0, \frac{1}{2}\right]\right)$ $=H\left(l_{j} \times\left[0, \frac{1}{2}\right]\right)$. The identity map $l_{j} \rightarrow l_{j}$ extends to a homeomorphism $E_{j} \rightarrow D_{j}$ which extends to homeomorphism $p_{j}: F\left(E_{j} \times[-j / n, 0]\right) \rightarrow H\left(D_{j} \times\left[0, \frac{1}{2}\right]\right)$ such that $p_{j}\left(F\left(l_{j} \times[-j / n, 0]\right)\right)=H\left(l_{j} \times\left[0, \frac{1}{2}\right]\right)$. The map $f_{j-1}: K_{j-1} \rightarrow L_{j-1}$ is defined to be $f_{j}$ on $K_{j}$ and $p_{j}$ on $F\left(E_{j} \times[-j / n, 0]\right)$. Thus, $f_{j-1}$ is a homeomorphism from $K_{j-1}$ onto $L_{j-1}$ such that if $1 \leqq i \leqq j-1$ then $f_{j-1}\left(F\left(l_{i} \times[-i / n, 0]\right)\right)=H\left(l_{i} \times\left[0, \frac{1}{2}\right]\right)$.

Now suppose, on the other hand, that $H\left(l_{j} \times[0,1]\right) \subset E_{j}$. Then $K_{j-1}=K_{j}$ $-F\left(E_{j} \times(-j / n, 0]\right)$ and $L_{j-1}=L_{j}$. It is evident from the proof of statement (4.3) that $F\left(E_{j} \times\left[-\left(j+\frac{1}{2}\right) / n, 0\right]\right) \subset K_{j}$, which implies that $K_{j-1} \cap F\left(E_{j} \times[-j / n, 0]\right)$ is the $\operatorname{disk} F\left(E_{j} \times\{-j / n\}\right)$. The set $F\left(l_{1} \times[-1 / n, 0]\right) \cup \cdots \cup F\left(l_{j-1} \times[-(j-1) / n, 0]\right)$ is closed and it is disjoint from $F\left(E_{j} \times[-j / n, 0]\right)$ because of the indexing criterion on the disks $E_{i}$. Thus, there is a homeomorphism $q_{j}: K_{j-1} \rightarrow K_{j}$ which is the identity on that set (see Theorem 6 on p. III-19 of Zeeman [9], for example). Define $f_{j-1}: K_{j-1} \rightarrow L_{j-1}$ to be the homeomorphism $f_{j} q_{j}$. The definition of $q_{j}$ assures one that if $1 \leqq i \leqq j-1$ then $f_{j-1}\left(F\left(l_{i} \times[-i / n, 0]\right)\right)=H\left(l_{i} \times\left[0, \frac{1}{2}\right]\right)$. Thus, statement (4.5) is proved.

Correspondingly, one also proves statement $(4.5)^{\prime}$. 
$(4.5)^{\prime}$ For $j=0, \ldots, m$, there is a homeomorphism $f_{j}^{\prime}: K_{j}^{\prime} \rightarrow L_{j}^{\prime}$. In particular, $K_{0}^{\prime}$ and $L_{0}^{\prime}$ are homeomorphic homotopy 3-cells.

(4.6) The homotopy 3-cells $L_{0}$ and $L_{0}^{\prime}$ are 3-cells.

Proof of (4.6). By the symmetry of the definitions of $L_{0}$ and $L_{0}^{\prime}$, it suffices to show that $L_{0}$ is a 3-cell. To this intent, let $D^{*}$ denote the disk $D_{0} \cup D_{1} \cup \cdots \cup D_{m}$. One observes that $D^{*}$ is the smallest subdisk of $D$ which contains the disk-withholes $D_{0}$. Also, let $L_{0}^{*}=L_{0}-H\left(D^{*} \times\left[0, \frac{1}{4}\right)\right)$, so $L_{0}^{*}$ is homeomorphic to $L_{0}$. The boundary of $L_{0}^{*}$ is in general position with respect to the disk $D$ and it has at least $m+1$ fewer components of intersection (representing the loops $l_{0}, \ldots, l_{m}$ ) with $D$ than bd $(C)$ has. By the induction hypothesis, $L_{0}^{*}$ is a 3-cell. Hence, so is $L_{0}$.

Since $K_{0} \approx L_{0}$ and $K_{0}^{\prime} \approx L_{0}^{\prime}$ and $K_{0} \cap K_{0}^{\prime}$ is the disk $R_{0}$, which is properly imbedded in $C$, and since $C=K_{0} \cup K_{0}^{\prime}$, the homotopy 3-cell $C$ is a 3-cell. Hence, the 3-manifold $B$ is in PC.

\section{Spheres and projective planes are $P$-admissible.}

THEOREM 2. Let $M$ be a 3-manifold (connected or not) in PC. Let $S^{\prime}$ and $S^{\prime \prime}$ be two distinct components of bd $(M)$ which are both spheres. And let $B$ be a 3-manifold obtained from $M$ be identifying $S^{\prime}$ and $S^{\prime \prime}$ under homeomorphism. Then $B$ is in PC.

Proof. Let $D^{\prime}$ and $D^{\prime \prime}$ be disks on $S^{\prime}$ and $S^{\prime \prime}$ respectively such that the given homeomorphism $S^{\prime} \rightarrow S^{\prime \prime}$ carries $D^{\prime}$ onto $D^{\prime \prime}$. Let $B^{\prime}$ be the 3-manifold obtained from $M$ by identifying $D^{\prime}$ and $D^{\prime \prime}$ under the restriction of the homeomorphism $S^{\prime} \rightarrow S^{\prime \prime}$. By Theorem $1, B^{\prime}$ is in PC. The 3-manifold $B^{\prime \prime}$ obtained from $B^{\prime}$ by filling in a 3-cell along the boundary component $\operatorname{cl}\left(S^{\prime}-D^{\prime}\right) \cup \operatorname{cl}\left(S^{\prime \prime}-D^{\prime \prime}\right)$ is homeomorphic to $B$. By Theorem 3 ("homogeneity") of Gugenheim [3], a homotopy 3 -cell can be imbedded in $B^{\prime \prime}$ only if it can be imbedded in $B^{\prime}$. Hence, $B^{\prime \prime}$ is in $\mathrm{PC}$ and, consequently, so is $B$.

TheOREM 3. Let $M$ be a 3-manifold (connected or not) in PC. Let $P^{\prime}$ and $P^{\prime \prime}$ be two distinct components of bd $(M)$ which are both projective planes. And let $B$ be a 3-manifold obtained from $M$ by identifying $P^{\prime}$ and $P^{\prime \prime}$ under a homeomorphism. Then $B$ is in $P C$.

Proof. Let the projective plane $P$ be the image in $B$ of the projective planes $P^{\prime}$ and $P^{\prime \prime}$. If a given homotopy 3 -cell can be imbedded in $B$, then there is an imbedding of that homotopy 3-cell such that its image $C$ lies in the interior of $B$ and such that the components of bd $(C) \cap P$ are simple loops, each a crossing of surfaces.

The first thing to be established is that each component of bd $(C) \cap P$ is a disk or a disk-with-holes. The alternative, of course, is that some component $R$ of bd $(C) \cap P$ is a nonorientable 2 -manifold. One observes that $R$ is properly imbedded in the homotopy 3-cell $C$, and that $R$ is a cycle in $C_{2}\left(C\right.$, bd $\left.(C) ; Z_{2}\right)$. Since the homology of $C$ is trivial, $R$ must be a boundary, which implies that $C$ contains a nonorientable 3-dimensional submanifold, which is a contradiction. 
Thus, it is established that each component of bd $(C) \cap P$ is a disk or a disk-withholes.

The next fact needed is that there is a disk on the projective plane $P$ which contains $C \cap P$ in its interior. Let $R_{1}, \ldots, R_{s}$ be the components of $C \cap P$. For $=1, \ldots, s$, it follows from the classification of compact 2-manifolds that exactly one component of $P$-int $\left(R_{j}\right)$ is a Möbius band and every other component is a disk. For $j=1, \ldots, s$, let $D_{j}$ be the disk which is the union of $R_{j}$ and all the components of $P$-int $\left(R_{j}\right)$ which are disks. Let $D$ be a disk on $P$ which contains $D_{1} \cup \ldots \cup D_{s}$ in its interior. Then the disk $D$ contains $C \cap P$ in its interior.

Now let $D^{\prime}$ and $D^{\prime \prime}$ be the pre-images of the disk $D$ in the projective planes $P^{\prime}$ and $P^{\prime \prime}$ respectively. The homotopy 3 -cell $C$ can evidently be imbedded in the 3-manifold $B^{\prime}$ obtained from $M$ by identifying $D^{\prime}$ and $D^{\prime \prime}$ under the restriction of the homeomorphism $P^{\prime} \rightarrow P^{\prime \prime}$. which is given in the hypothesis. By Theorem 1 , the 3-manifold $B^{\prime}$ is in PC. Hence, $C$ is a 3-cell. Hence, the 3-manifold $B$ is in PC.

6. $P$-admissibility and the Poincaré conjecture. The first statement of the Pasting Theorem of $\S 3$ has been established via Theorems 1, 2, and 3. The second statement of the Pasting Theorem will be proved in this section as Theorems 4 and 5.

Let $B$ be a 3-manifold in PC and let $R$ be a compact connected 2-dimensional submanifold of bd $(B)$. One says that $(B, R)$ is a $P$-admissible pair if the following holds: For any 3-manifold $B^{\prime}$ (disjoint from $B$ ) in PC and for any imbedding $f: R \rightarrow$ bd $\left(B^{\prime}\right)$, the 3-manifold obtained from $B \cup B^{\prime}$ by identifying, for each point $x$ in $R$, the points $x$ and $f(x)$ in bd $\left(B \cup B^{\prime}\right)$ is in PC.

A solid torus is a 3-manifold which is homeomorphic to the product of a disk and a 1-sphere.

Lemma 1. Let $T$ be a solid torus. If the pair $(T$, bd $(T))$ is $P$-admissible, then the Poincaré conjecture is true.

Proof. Let $N$ be any compact, orientable, connected 3-manifold with vacuous boundary. By Theorem 6 of Wallace [7], a homeomorphic copy of $N$ can be constructed by removing from the 3-sphere $S^{3}$ a collection of disjoint solid tori and sewing them back differently. The closure of the complement in $S^{3}$ of the union of the solid tori is in PC because it is a submanifold of $S^{3}$. If the pair $(T$, bd $(T)$ ) is $P$-admissible, then any 3 -manifold which results from sewing the tori back is in PC. Thus, every compact, orientable, connected 3-manifold with vacuous boundary would be in PC. This would imply, of course, that the Poincare conjecture is true.

Lemma 2. Let $K$ be a 3-cell and let $A$ be an annulus (i.e. $\left.A \approx S^{1} \times[0,1]\right)$ on bd $(K)$. If the pair $(K, A)$ is $P$-admissible, then the Poincaré conjecture is true.

Proof. Let $B$ be a 3-manifold in PC which has a boundary component $R$ such that $R$ is a torus. Let $T$ be a solid torus disjoint from $B$ and $h:$ bd $(T) \rightarrow R$ a 
homeomorphism. It will be shown that if $(K, A)$ is $P$-admissible, then the 3-manifold $B_{1}$ obtained from $B \cup T$ by identifying bd $(T)$ and $R$ under $h$ is in PC, which implies that $(T$, bd $(T))$ is a $P$-admissible pair, which implies by Lemma 1 that the Poincaré conjecture is true.

Suppose that $(K, A)$ is $P$-admissible. Let $g: K \rightarrow T$ be an imbedding such that $g(K) \cap$ bd $(T)=g(A)$ and $\mathrm{cl}(T-g(K))$ is a 3-cell. Since $(K, A)$ is $P$-admissible, the 3-manifold $B_{2}$ obtained from $B \cup g(K)$ by identifying $g(A)$ and $h g(A)$ via $h$ is in PC. The 3-manifold $B_{3}$ obtained from $B_{2}$ by filling in a 3-cell along the 2-sphere $(R-h g(A)) \cup h g($ bd $(K)-\operatorname{int}(A))$ is in PC, by Theorem 3 of Gugenheim [3]. Since $B_{1}$ and $B_{3}$ are homeomorphic, $B_{1}$ is in PC, which implies that the Poincaré conjecture is true.

THEOREM 4. If any orientable 2-manifold which is neither a disk nor a sphere is $P$-admissible, then the Poincaré conjecture is true.

Proof. Statements (6.1) and (6.2) are intermediate results.

(6.1) Let $V$ and $W$ be orientable compact connected 2-manifolds whose boundaries have the same number of components and such that genus $(W)=\operatorname{genus}(V)+1$. Let $W$ be $P$-admissible. Then $V$ is $P$-admissible.

Proof of (6.1). Let $M$ be a 3-manifold in PC. Let $V^{\prime}$ and $V^{\prime \prime}$ be two disjoint homeomorphic copies of $V$ on bd $(M)$. And let $h: V^{\prime} \rightarrow V^{\prime \prime}$ be a homeomorphism. It will be shown that the 3-manifold $B$ obtained by identifying $V^{\prime}$ and $V^{\prime \prime}$ under $h$ is in PC.

Let $T$ be a solid torus in $M$ whose intersection with bd $(M)$ is a disk in the nterior of $V^{\prime}$ (see Figure 4a). Let $U$ be a solid torus which is disjoint from $M$, and let $g: T \rightarrow U$ be a homeomorphism. Let $M_{1}$ be the 3-manifold obtained from $\mathrm{cl}(M-T)$ by pasting the solid torus $U$ to $\mathrm{cl}(M-T)$ at the disk $h(T \cap$ bd $(M))$ via the composition of $h$ restricted to $T \cap$ bd $(M)$ and $g^{-1}$ restricted to the disk $g(T \cap$ bd $(M))$ on bd $(U)$ (see Figure $4 \mathrm{~b})$.

Since $\mathrm{cl}(M-T) \subset M, \mathrm{cl}(M-T)$ is in PC. By Theorem 1, the 3-manifold $M_{1}$ is in PC. Let $W^{\prime}=\left(V^{\prime} \cup\right.$ bd $\left.(T)\right)-$ int $\left(T \cap V^{\prime}\right)$ and $W^{\prime \prime}=\left(V^{\prime \prime} \cup\right.$ bd $\left.(U)\right)$
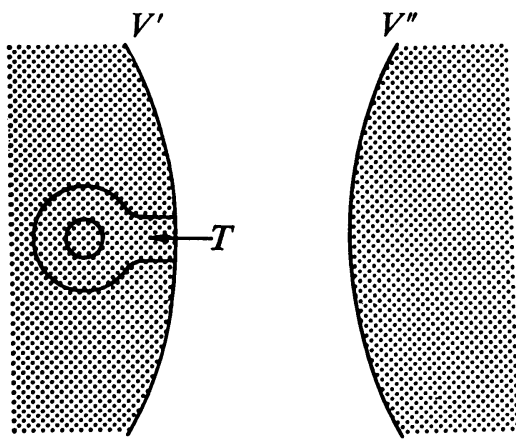

FIGURE 4a

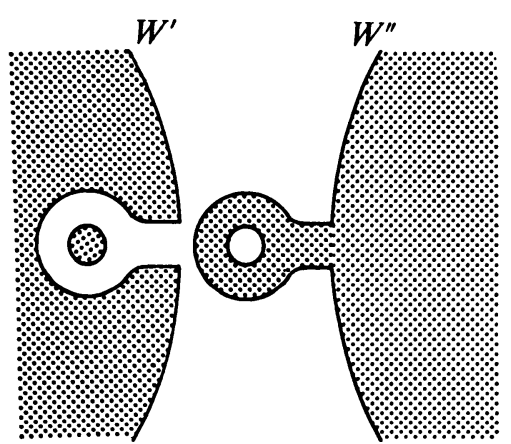

Figure 4b 
-int $\left(h\left(T \cap V^{\prime}\right)\right)$. So $W^{\prime}$ and $W^{\prime \prime}$ are homeomorphic to the $P$-admissible 2-manifold $W$. Thus, the 3-manifold $M_{2}$ which is obtained from $M_{1}$ by identifying $W^{\prime}$ - bd $(T)=V^{\prime}-$ bd $(T)$ with $W^{\prime \prime}-$ bd $(U)=V^{\prime \prime}-h($ bd $(T))$ via $h$ and by identifying $W^{\prime} \cap$ bd $(T)=$ bd $(T)-V^{\prime}$ with $W^{\prime \prime} \cap$ bd $(U)=h\left(\mathrm{bd}(T)-V^{\prime \prime}\right)$ under $g$ is in PC. Evidently, $B$ is homeomorphic to $M_{2}$. Hence, the 3-manifold $B$ is in PC, which proves that the 2-manifold $V$ is $P$-admissible.

(6.2) Let $V$ and $W$ be orientable compact connected 2-manifolds which have the same genus and such that bd $(W)$ has one more component than bd $(V)$. Let $W$ be $P$-admissible. Then $V$ is $P$-admissible.

The proof of statement (6.2) is omitted, since it is an easy generalization of the proof of Theorem 2. The Poincaré conjecture is true if the torus is $P$-admissible, by Lemma 1 . By statement (6.1), it is true if any compact orientable 2-manifold with positive genus and vacuous boundary is $P$-admissible. In fact, by statement (6.2), it is also true if any compact orientable 2-manifold with positive genus and nonvacuous boundary is $P$-admissible. The Poincaré conjecture is true if the annulus is $P$-admissible, by Lemma 2 . By statement (6.2), it is true if any disk-withholes is $P$-admissible. This completes the proof of Theorem 4.

Lemma 3. Let $R$ be a Möbius band. Then the pair $(R \times[0,1]$, bd $(R) \times[0,1])$ is $P$-admissible.

Proof. Let $M$ be a 3-manifold in PC and let $h$ : bd $(R) \times[0,1] \rightarrow$ bd $(M)$ be an imbedding. It will be shown that the 3-manifold $B$ obtained from $M \cup(R \times[0,1])$ by identifying the domain and the image of $h$ is in PC. Let $k$ be an orientation reversing loop on $R$ and let $A$ be the annulus $k \times[0,1]$ in $R \times[0,1]$. If a given homotopy 3-cell can be imbedded in $B$, then there is an imbedding of that homotopy 3-cell such that its image $C$ lies in the interior of $B$ and such that the components of bd $(C) \cap A$ are simple loops, each a crossing of surfaces.

Each component $S$ of bd $(C) \cap A$ is orientable, for homological reasons given in the proof of Theorem 3, so it must be a disk or a disk-with-holes. None of the boundary components of $S$ can separate $k \times 0$ from $k \times 1$ because then $k \times 0$ and $k \times 1$ would be freely homotopic to that boundary component of $S$. But each component of bd $(S)$ is trivial, because $S$ lies in the homotopy 3-cell $C$. This would imply that $k \times 0$ and $k \times 1$ are trivial in $B$, which is a contradiction. It follows that $S$ lies on a disk which is a submanifold of the annulus $A$.

Let $T$ be a tubular neighborhood of the loop $k$ in the interior of the Möbius band $R$, chosen so that the components of bd $(C) \cap(b d(T) \times[0,1])$ are simple loops, each a crossing of surfaces. One observes that $T$ is a Möbius band and that $\operatorname{cl}(B-(T \times[0,1]))$ is homeomorphic to the 3-manifold $M$ and, therefore, in PC. Since each component of $C \cap(b d(T) \times[0,1])$ lies on a disk on the annulus bd $(T) \times[0,1]$, one may prove that $C$ is a 3 -cell by applying Theorem 1 . That is, take $D$ to be a disk in the interior of bd $(T) \times[0,1]$ such that $C \cap(b d(T) \times[0,1])$ lies in the interior of $D$. Then $C$ lies in the 3-manifold $B^{\prime}$ obtained from 
cl $(B-(T \times[0,1]))$ and $T \times[0,1]$ by cutting $B$ across $($ bd $(T) \times[0,1])-D$. By Theorem $1, B^{\prime}$ is in PC.

THEOREM 5. If any nonorientable 2-manifold which is not a projective plane is $P$-admissible, then the Poincaré conjecture is true.

Proof. The following statement is the main fact needed.

(6.3) Let $V$ and $W$ be compact connected 2-manifolds such that one can obtain a homeomorphic copy of $V$ by removing the interior of a Möbius band from $W$. Let $W$ be $P$-admissible. Then $V$ is $P$-admissible.

Proof of (6.3). Let $M$ be a 3-manifold in PC. Let $V^{\prime}$ and $V^{\prime \prime}$ be two disjoint homeomorphic copies of $V$ on bd $(M)$. And let $h: V^{\prime} \rightarrow V^{\prime \prime}$ be a homeomorphism. It will be shown that the 3-manifold $B$ obtained by identifying $V^{\prime}$ and $V^{\prime \prime}$ under $h$ is in PC.

Let $k$ be a component of bd $\left(V^{\prime}\right)$ and let $A$ be a regular neighborhood of the loop $k$ in cl (bd $\left.(M)-V^{\prime}\right)-V^{\prime \prime}$ such that $h(A) \cap V^{\prime}$ is empty. Let $R$ be a Möbius band. And let $M_{1}$ be the 3-manifold obtained from $M \cup(R \times[0,1]) \cup(R \times[2,3])$ by identifying the annulus $A$ and bd $(R) \times[0,1]$ under a homeomorphism which takes bd $(R) \times\{0\}$ onto the loop $k$ and by identifying the annulus $h(A)$ and bd $(R) \times[2,3]$ under a homeomorphism which takes bd $(R) \times\{2\}$ onto the loop $h(k)$ (see Figure 5).

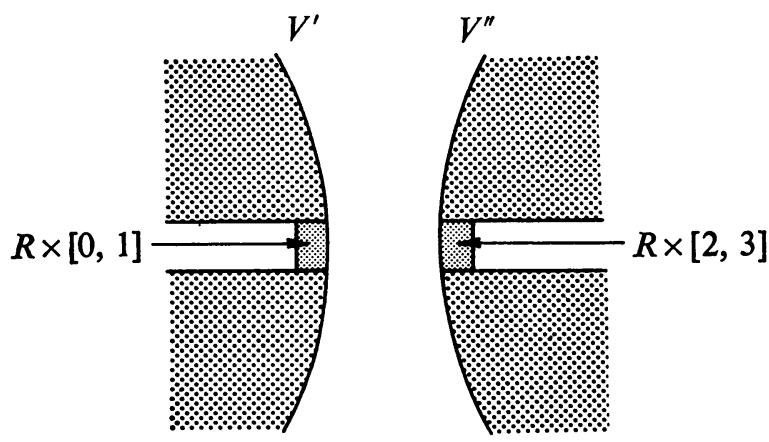

FIGURE 5

The 3-manifold $M_{1}$ is in PC, because of Lemma 3. Let $W^{\prime}=V^{\prime} \cup(R \times\{0\})$ and let $W^{\prime \prime}=V^{\prime \prime} \cup(R \times\{2\})$. Then $W^{\prime}$ and $W^{\prime \prime}$ are homeomorphic to the 2-manifold $W$. Let $f: W^{\prime} \rightarrow W^{\prime \prime}$ be a homeomorphism which agrees with $h: V^{\prime} \rightarrow V^{\prime \prime}$ on $V^{\prime}$. Then let $B_{1}$ be the 3-manifold obtained from $M_{1}$ by identifying $W^{\prime}$ and $W^{\prime \prime}$ under $f$. Since $W$ is $P$-admissible, $B_{1}$ is in PC. Since $B \subset B_{1}$, it follows that $B$ is in PC. Hence, $B$ is $P$-admissible.

The proof of Theorem 5 is now easily completed. Let $Q$ be any nonorientable, compact, connected 2-manifold. If $m$ is the number of crosscaps on $Q$ and $r$ is the number of components of bd $(Q)$, it follows from $m$ applications of statement (6.3) 
that $Q$ is $P$-admissible only if the disk-with- $(m+r-1)$-holes is $P$-admissible. By Theorem 4, therefore, if any nonorientable compact connected surface other than a projective plane is $P$-admissible, the Poincaré conjecture is true.

7. Added in proof. Let $M$ be a 3-manifold, let $R$ be a compact, connected 2dimensional submanifold of bd $(M)$, and suppose that the component $M^{\prime}$ of $M$ which contains $R$ is not a 3-cell. One says that $R$ is incompressible if the kernel of the induced map $\pi_{1}(R) \rightarrow \pi_{1}\left(M^{\prime}\right)$ is trivial. C. D. Feustel and the author have jointly observed that the proof of Theorem 1 can be easily modified to give a proof of the following theorem.

THEOREM 6. Let $M$ be a 3-manifold (connected or not) in PC. Let $R^{\prime}$ and $R^{\prime \prime}$ be disjoint, homeomorphic, incompressible surfaces in bd $(M)$, and let $B$ be a 3-manifold obtained from $M$ by identifying $R^{\prime}$ and $R^{\prime \prime}$ under a homeomorphism. Then $B$ is in PC.

\section{REFERENCES}

1. E. M. Brown, Unknotting in $M^{2} \times I$, Trans. Amer. Math. Soc. 123 (1966), 480-505.

2. J. L. Gross, $A$ unique decomposition theorem for 3-manifolds with connected boundary, Trans. Amer. Math. Soc. 142 (1969), 191-199.

3. V. K. A. M. Gugenheim, Piecewise linear isotopy and embedding of elements and spheres. I, Proc. London Math. Soc. 3 (1953), 29-53.

4. P. J. Hilton and S. Wylie, Homology theory, Cambridge Univ. Press, New York, 1960.

5. J. Milnor, A unique decomposition theorem for 3-manifolds, Amer. J. Math. 84 (1962), 1-7.

6. E. E. Moise, Affine structures in 3-manifolds. V, Ann. of Math. (2) 56 (1952), 96-114.

7. A. H. Wallace, Modifications and cobounding manifolds, Canad. J. Math. 12 (1960), 503-528.

8. J. H. C. Whitehead, Simplicial spaces, nuclei, and m-groups, Proc. London Math. Soc. 45 (1939), 243-327.

9. E. C. Zeeman, Seminar on combinatorial topology, Mimeographed Notes, Inst. Hautes Études Sci. Publ. Math., 1963.

Dartmouth College, HANOVER, New HampshiRe 